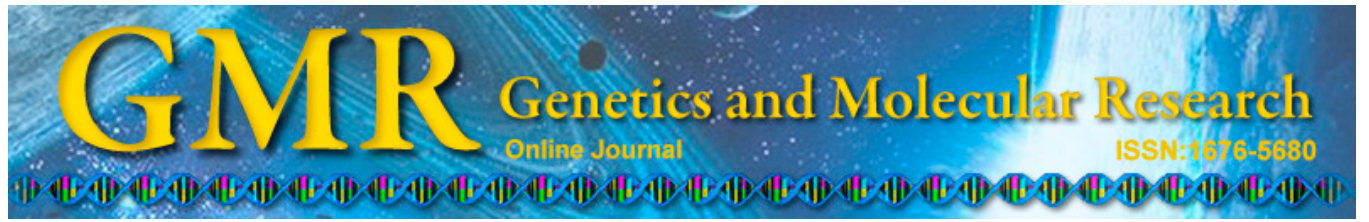

\title{
Characterization of MUSTN1 gene and its relationship with skeletal muscle development at postnatal stages in Pekin ducks
}

\author{
T.S. Xu ${ }^{1,2,3}$, L.H. Gu ${ }^{1}$, Y. Sun ${ }^{5}$, X.H. Zhang ${ }^{4}$, B.G. Ye ${ }^{6}$, X.L. Liu ${ }^{1}$ and S.S. Hou ${ }^{3}$ \\ ${ }^{1}$ Shaanxi Key Laboratory of Molecular Biology for Agriculture, \\ College of Animal Science and Technology, Northwest A\&F University, \\ Yangling, Shaanxi, China \\ ${ }^{2}$ Tropical Crops Genetic Resources Research Institute, \\ Chinese Academy of Tropical Agricultural Sciences, Danzhou, Hainan, China \\ ${ }^{3}$ Institute of Animal Sciences, Chinese Academy of Agricultural Science, \\ Beijing, China \\ ${ }^{4}$ College of Animal Science, Henan University of Science and Technology, \\ Luoyang, He'nan, China \\ ${ }^{5}$ Institute of Animal Science \& Veterinary, \\ Hainan Academy of Agriculture Science, Haikou, Hainan, China \\ ${ }^{6}$ Animal Breeding and Genomics Centre, Wageningen University, \\ Wageningen, The Netherlands \\ Corresponding authors: X.L. Liu / S.S. Hou \\ E-mail: xiaolinliu2010@126.com / houss2010@126.com
}

Genet. Mol. Res. 14 (2): 4448-4460 (2015)

Received October 15, 2014

Accepted December 11, 2014

Published May 4, 2015

DOI http://dx.doi.org/10.4238/2015.May.4.2

\begin{abstract}
Musculoskeletal embryonic nuclear protein 1 (MUSTN1) gene is involved in myogenic fusion and differentiation in rats. We previously showed the differential expression of MUSTN1 in week (W) 2 and W6 breast muscles of Pekin ducks. In this study, we further investigated its molecular characteristics and expression profiles in different tissues at $\mathrm{W} 7$ and in breast and leg muscles at W1, W3, W5, W7, and W9. The relationship between muscle development and
\end{abstract}


muscle fiber areas was also investigated. A 358-bp cDNA sequence was obtained. The coding sequence of duck MUSTN1 cDNA encoded a 78-amino acid sequence, which showed high similarity with those of other species (96\% similarity with zebra finch and $94 \%$ with chicken). In addition, a 6435-bp genomic DNA sequence of MUSTN1 was obtained. In total, 231 transcription factor-binding sites were found in the promoter region, and many of these transcription factors were involved in the regulation of muscle development. MUSTN1 expression in breast muscle increased from $\mathrm{W} 1$ to $\mathrm{W} 5$ and then decreased at W9. In leg muscle, the expression increased from $\mathrm{W} 1$ to $\mathrm{W} 3$ and then decreased. The relative growth rates of breast and leg muscle fibers reached their peaks at W3-W5 and W1-W3, respectively. Since the greatest relative growth rates appeared at the highest expression levels of the MUSTN1 gene, it was thought to play roles in duck muscle development. Our findings would be helpful in understanding the molecular characteristics and functions of the MUSTN1 gene in breast muscle development of ducks.

Key words: MUSTN1; Pekin ducks; Skeletal muscle development; Molecular characteristics; Expression profiles

\section{INTRODUCTION}

Muscle development is a complicated process that includes somatic cell proliferation, migration, and differentiation; the muscle fibers are formed during embryonic development, and they elongate and enlarge in the postnatal stage. Thousands of genes are associated with muscle development, such as myogenic differentiation antigen $(M y o D)$ family (Kanisicak et al., 2009; Li et al., 2010; Liu et al., 2011), myocyte enhancer factor 2 (MEF2) family (Maiti et al., 2008), paired box gene 3 (Pax3; Buckingham et al., 2003), growth hormone-releasing hormone (GHRH; Draghia-Akli et al., 2002), insulin-like growth factor 1 (IGF1; Wu et al., 2009; Chandra et al., 2011), and myostatin (MSTN; Lee and McPherron, 1999; Gu et al., 2004). Although many studies have investigated the mechanisms underlying muscle development, many aspects of this process are not yet known.

Musculoskeletal embryonic nuclear protein 1 (MUSTN1) gene, which was identified in 2002 in rats, encodes a small nuclear protein of 82 amino acids (Hadjiargyrou et al., 2002; Lombardo et al., 2004). In normal adult rat tissues, MUSTN1 expression is predominantly detected in skeletal muscles and tendons (Liu et al., 2010). Silencing of rat MUSTN1 leads to the inhibition of myogenic fusion and differentiation, indicating its role in muscle development (Liu et al., 2010). Characterization of the MUSTN1 promoter revealed 4 AP-1 domains, one of which is responsible for substantial transcriptional activation. c-Fos, Fra-2, and JunD members of AP-1 family - are required for the transcriptional activation of MUSTN1 (Liu and Hadjiargyrou, 2006). Previous studies indicated that MUSTN1 represents an important factor in the development of skeletal muscles.

In our previous study, the genes that were differentially expressed in Pekin duck breast muscles between weeks 2 and 6 were identified using suppression subtractive hybridization technique. The level of MUSTN1 mRNA varied significantly between the 2 stages, suggesting 
that it is a potential candidate for muscle development (Xu et al., 2012). In this study, the fulllength cDNA and entire genomic DNA sequence of the duck MUSTN1 gene were cloned. The spatial and temporal expression patterns of the gene were analyzed. Furthermore, the relationship of the expression patterns of the gene with the cross-section areas of Pekin duck breast and leg muscle fibers was also investigated. The results of this study might help understand the molecular characteristics of the MUSTN1 gene and its relationship with skeletal muscle development.

\section{MATERIAL AND METHODS}

\section{Ethics statement}

This study was performed according to the Guide for the Care and Use of Agricultural Animals in Research and Teaching (Federation of Animal Science Societies, 2010). Bird slaughtering and transport were humanely performed as necessary to ameliorate their suffering.

\section{Bird and tissue collection}

One hundred 1-day-old male Z5 Pekin ducks (lean-type), from the breeding farm of Z-type Pekin ducks of the Institute of Animal Sciences, Chinese Academy of Agricultural Science (Beijing, China), were randomly allocated to 10 pens with 10 ducks per pen. Experimental ducks were kept in plastic-wire-floor pens in an environmentally controlled duck house; the size of each pen was $100 \times 100 \times 40 \mathrm{~cm}$. The temperature was set at $28^{\circ} \mathrm{C}$ from 1 to 3 days of age; $26^{\circ} \mathrm{C}$, from 4 to 7 days of age; $25^{\circ} \mathrm{C}$, from 8 to 14 days of age; $22^{\circ}$ to $20^{\circ} \mathrm{C}$, from 15 to 21 days of age; and room temperature, from 22 to 63 days of age. All ducks had free access to pelleted feed and water. Five healthy and moderate sized ducks were selected at 1, 3, 5,7 , and 9 weeks of age (denoted as $1 \mathrm{~W}, 3 \mathrm{~W}, 5 \mathrm{~W}, 7 \mathrm{~W}$, and $9 \mathrm{~W}$, respectively) to collect breast muscle (BM; from pectoralis major) and leg muscle (LM; from thigh) samples. Each BM or LM sample was separated into 2 pieces. One was dipped in $4 \%$ formalin for $24-48 \mathrm{~h}$ at room temperature and then stored at $-80^{\circ} \mathrm{C}$ for paraffin sectioning and morphologic analysis. The other was immediately immersed in liquid nitrogen and stored at $-80^{\circ} \mathrm{C}$ until further qualitative reverse transcriptase-polymerase chain reaction (qRT-PCR) analysis. At 7 weeks of age, blood samples from wing vein of ducks were collected into $\mathrm{Na}_{2}$ EDTA-containing tubes and kept at $-20^{\circ} \mathrm{C}$ until genomic DNA isolation. Pieces of heart, liver, gizzard, small intestine, abdominal fat, and tibia were collected after exsanguination and immediately immersed in liquid nitrogen until RNA isolation for tissue-specific expression analysis.

\section{Total RNA isolation, cDNA synthesis, and genomic DNA isolation}

Total RNA was isolated from all tissue samples by using RNAiso Plus kit (Takara, Dalian, China) following manufacturer instructions. The RNA quality was analyzed using $1.0 \%$ agarose gel electrophoresis and spectrophotometric absorption measurement at $260 \mathrm{~nm}$ by using a Nanodrop ND- $1000^{\circledR}$ Spectrophotometer. All RNA samples were treated with DNase I recombinant (Roche, Shanghai, China) and reversely transcribed to cDNA by using PrimeScript $^{\mathrm{TM}} \mathrm{RT}$ reagent Kit (Takara) following manufacturer instructions. The genomic DNA was isolated from blood samples according to Sambrook and Russell (2001). 


\section{Cloning, sequencing, and analysis of MUSTN1}

Seven pairs of primers to clone MUSTN1 from the genomic DNA were designed using the Primer 5 software and synthesized by the Sangon Biological Engineering Technology Company (Shanghai, China; Table 1 - primers pMUSTN-1-7). Primer pMUSTN-8 was used to clone the cDNA sequence of MUSTN1. The PCR products were cloned into pMD-19T plasmid vector (Takara) according to manufacturer instructions and transformed into Escherichia coli DH5 . The positive clones were sequenced by the Sangon Biological Engineering Technology Company.

\begin{tabular}{|c|c|c|c|}
\hline Primer & Sequence (5' to $3^{\prime}$ ) & $\operatorname{Tm}\left({ }^{\circ} \mathrm{C}\right)$ & Product (bp) \\
\hline pMUSTN-1 & F TTGAAGCAAAGCCAGACC & 55.02 & 1227 \\
\hline pMUSTN-2 & $\begin{array}{l}\text { R AAGACTACCAGAGTGGAAAG } \\
\text { F CTGTCTTGTGGAAATCGC }\end{array}$ & $\begin{array}{l}55.75 \\
55.02\end{array}$ & 893 \\
\hline & R CCCACTGTATGTGCTGAC & 57.30 & \\
\hline pMUSTN-3 & $\begin{array}{l}\text { F TAAAAGGGGGGAAACAGC } \\
\text { R GTAACCTGCTTGAACGAGAG }\end{array}$ & $\begin{array}{l}55.02 \\
57.80\end{array}$ & 986 \\
\hline pMUSTN-4 & $\begin{array}{l}\text { F GATTTCGGGCGAGTGAGC } \\
\text { R GACTTCGGCATAGTTGTGGC }\end{array}$ & $\begin{array}{l}59.58 \\
59.85\end{array}$ & 1156 \\
\hline pMUSTN-5 & $\begin{array}{l}\text { F TGACAGCGAGCTATTCCG } \\
\text { R AACCACAACTCCACATCAGG }\end{array}$ & $\begin{array}{l}57.30 \\
57.80\end{array}$ & 1045 \\
\hline pMUSTN-6 & $\begin{array}{l}\text { F GCTGGGCAATCAGAAGAC } \\
\text { R GCATCTGTAGGTGGTCGTC }\end{array}$ & $\begin{array}{l}59.72 \\
57.30\end{array}$ & 1019 \\
\hline pMUSTN-7 & $\begin{array}{l}\text { F AAGGAACTTGGGGTGGTC } \\
\text { R CAGCGAGGAGTTGTTTGG }\end{array}$ & $\begin{array}{l}55.69 \\
58.05\end{array}$ & 1401 \\
\hline pMUSTN-8 & $\begin{array}{l}\text { F AAGGATTTCGGGCGAGTG } \\
\text { R CAGTGAGGTTAGTGTAAGGGG }\end{array}$ & $\begin{array}{l}55.60 \\
56.74\end{array}$ & 345 \\
\hline pMUSTN-9 & $\begin{array}{l}\text { F AAAAAGAAGCGTCCTCC } \\
\text { R AAGACTGTTTCACCTCCTG }\end{array}$ & $\begin{array}{l}52.18 \\
55.41\end{array}$ & 173 \\
\hline$\beta$-actin & $\begin{array}{l}\text { F GCTATGTCGCCCTGGATTTC } \\
\text { R CACAGGACTCCATACCCAAGAA }\end{array}$ & $\begin{array}{l}55.50 \\
57.30\end{array}$ & 168 \\
\hline
\end{tabular}

Primers pMUSTN-1-7 were used to clone MUSTN1 from the genome DNA, primer pMUSTN-8 was used to clone the cDNA sequence of MUSTN1, primer pMUSTN-9 was used in qRT-PCR of the MUSTN1 gene, and primer $\beta$-actin is the primer of housekeeping gene $\beta$-actin.

The similarity of amino acid sequences of duck MUSTN1 with those of other species was analyzed using BLASTn (NCBI), and the coding sequence (CDS) region of duck cDNA sequences was predicted using Editseq in DNAstar. The different fragments of duck MUSTN1 DNA sequence were aligned using MegAlign in DNAstar. Phylogenetic tree was constructed using the MEGA 5.0 software by the neighbor-joining method and bootstrap sampling 1000 times. Transcription factor-binding sites in the putative promoter of MUSTN1 genome sequence were analyzed using the online JASPAR database (http://jaspar. genereg.net/).

\section{qRT-PCR analysis}

The SYBR PrimeScript RT-PCR kit (TaKaRa) and a reference gene ( $\beta$-actin) were used for detecting the expression level of MUSTN1. The qRT-PCRs were carried out using an iCycler IQ5 multicolor real-time PCR detection system (Bio-Rad, USA). The qRT-PCR 
contained $1 \mu \mathrm{L}$ cDNA template, $12.5 \mu \mathrm{L}$ SYBR Premix ExTaq, $9.5 \mu \mathrm{L}$ sterile water, and $1 \mu \mathrm{L}$ of each gene-specific primer (Table 1 - pMUSTN-9 or $\beta$-actin). Thermal cycling parameters were 1 cycle at $95^{\circ} \mathrm{C}$ for $2 \mathrm{~min}, 40$ cycles of $95^{\circ} \mathrm{C}$ for $15 \mathrm{~s}$, and $60^{\circ} \mathrm{C}$ for $34 \mathrm{~s}$. Dissociation curve analysis was performed after each real-time reaction to ensure that there was only one product. qRT-PCR analysis of each sample was repeated 3 times.

\section{Paraffin sectioning and morphologic analysis of muscle fibers}

$\mathrm{BM}$ and LM tissues were washed with running water and dehydrated in serially diluted ethanol, i.e., $75 \%$ for 4 h, $85 \%$ for 4 h, $95 \%$ overnight, and $100 \%$ for 2 h. Dehydrated tissues were treated with xylene 3 times and embedded in paraffin blocks, trimmed, and cut at $5 \mu \mathrm{m}$ by using Leica RM2235 (Germany). The paraffin ribbon was placed in a water bath at about $37^{\circ} \mathrm{C}$. Sections were mounted onto slides, dried in air for $30 \mathrm{~min}$, and then baked at $45^{\circ} \mathrm{C}$ in an oven overnight. Sections were dewaxed with xylene twice for $10 \mathrm{~min}$ each and hydrated in 2 changes of $100 \%$ ethanol for 3 min each, 95 and $80 \%$ ethanol for 1 min each, and finally, rinsed in distilled water for $5 \mathrm{~min}$. Slices were stained with hematoxylin-eosin (HE). Digital microscope (Axio Imager Z2; Germany) was used to observe and analyze sections. The average area of 200 muscle fibers from each sample was measured and calculated using the Axioversion 40 software. The relative growth rate (RGR) was defined as the increased area of muscle fibers from $\mathrm{n}-2$ weeks of age to $\mathrm{n}$ weeks of age. The equation is $R G R=\left(V_{n}-V_{n-2}\right) / V_{n-2}$, where $V_{n}$ is the average area of the muscle fiber from BM or LM of $\mathrm{n}$ weeks of age, and $V_{n-2}$ is the average area of the muscle fibers from BM or LM of $\mathrm{n}-2$ weeks of age.

\section{Statistical analysis}

The relative gene expression level was determined using the comparative cycle threshold (Ct) method (Livak and Schmittgen, 2001). The $\Delta \mathrm{Ct}$ value was calculated by subtracting the target $\mathrm{Ct}$ of each sample from its $\beta$-actin $\mathrm{Ct}$ value. Gene expression between different stages was analyzed by one-way analysis of variance (ANOVA), followed by the Bonferroni test for pairwise comparison to identify the difference in significance of gene expressions at various stages.

The unpaired $t$-test was used to compare the average area and RGR of the muscle fibers between BM and LM (SPSS for Windows, version 13.0, Chicago, IL, USA).

\section{RESULTS}

\section{Molecular cloning and sequence analysis of MUSTN1}

The cDNA sequence (358 bp) of the duck MUSTN1 gene (accession No. JX843529) was cloned from the BM of ducks. The cDNA sequence contained 5'-UTR (37 bp), CDS (237 bp), and 3'-UTR ( 84 bp; Figure 1). The CDS encoded a 78-amino acid sequence. This amino acid sequence showed high similarity with those of other species $(96 \%$ similarity with zebra finch and 94\% with chicken; Table 2). The typical start codon (ATG) and stop codon (TAG) were found in the cDNA (Figure 1). 


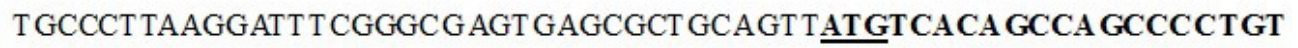 GAAAAA GA AGCGTCCTCCA GTGAA GGAAGAA GATCTCAA AGGA GCTA GA GGAAA TCTTTCCAAAAACCA GGAAATTAAATCTAAAACC TACCAA GTCATGAAGCA GT GT GAACAAATGGGCTCTGCA GCACCTTCCATATTCA GCCGA GATCGGACA GGA GGT GAAACA GTCTTTGA GAAACC TAAA GATGAA CCA GCCAAAA GCGTCTTTGGTTGAC AGCTGAGCT GAAAGTG AT TGT T GAGAT GAT T GTT TTAAT AT T T TCCCAGTT T TCTACCCC TTACACTAACCTCACT GAAGGGC}

Figure 1. cDNA sequence ( $358 \mathrm{bp}$ ) of duck MUSTN1. The sequence marked in bold ( $237 \mathrm{bp}$ ) is the CDS of the MUSTN1 gene, and the underlined codons (ATG and TGA) are start and stop codons, respectively. The sequence before the CDS is 5'-UTR (37 bp), and that after the CDS is 3'-UTR ( $84 \mathrm{bp}$ ).

\begin{tabular}{|c|c|c|}
\hline Accession No. & Species & Max ident \\
\hline XP_002193912.1 & Zebra finch [Taeniopygia guttata] & $96 \%$ \\
\hline NP_-998745.1 & Chicken [Gallus gallus] & $94 \%$ \\
\hline AD̄̄18076.1 & Pig $[$ Sus scrofa $]$ & $85 \%$ \\
\hline NP_001035679.1 & Cattle $[$ Bos taurus $]$ & $83 \%$ \\
\hline NP_995325.3 & Human [Homo sapiens] & $83 \%$ \\
\hline NP_-852033.1 & Rat $[$ Rattus norvegicus $]$ & $86 \%$ \\
\hline NP_852055.1 & Mouse [Mus musculus] & $81 \%$ \\
\hline
\end{tabular}

The MUSTN1 amino acid sequence also included a classic nuclear import signal (PVKKKRPPV, amino acids 6-14; Figure 2). The phylogenetic tree constructed on the basis of vertebrate MUSTN1 amino acid sequences showed that the sequences formed 3 main groups: avian MUSTN1 (duck, zebra finch, and chicken), murine MUSTN1 (rat and mouse), and human MUSTN1 (human and northern white-cheeked gibbon; Figure 3).

\begin{tabular}{|c|c|c|}
\hline \\
\hline \multicolumn{3}{|c|}{$\begin{array}{l}\text { Northern white-cheeked gib bon } \\
\qquad 1 \mathrm{msq} \text { agaqeapikkkrppvk }\end{array}$} \\
\hline Cattle: & 1 msqagaqeapikkkrppvkeedlkgargnltknqeikskty qvmreceqagstapsvfsrartg aetv fekpkagpaksvfg & 82 \\
\hline Human: & 1 msqagaqeapikkkrppvkeedlkg argnltknq eikskty qvmrec eqag saap svfsrtrtgtetvfekpkagptksvfg & 82 \\
\hline Rat: & 1 mseagtp eapikkkrppvkeed lkgargslsknqeikskty qvmrdyeqag saap sifsr nrtgtetvfekpkegpaksvfg & 82 \\
\hline Mouse: & 1 mseagtp egpikkkrppvkeedlkg argtlaknqdiksktyqvmrdyeqags aap svfsr nrtgtetvfekpkegpaksvfg & 82 \\
\hline Pig : & 1 msqeapikkkrppakeedlkgargnlaknqeikskty qvmreceqags aap svfsrartgtetvfekpkagp aksvfg & 78 \\
\hline Chicken: & 1 msqpdpvkkkrppvkeedlkgargnlsknqeiksktyqvmkqceqmgsaapsifsrartgsetvfekskdeppksvfg & 78 \\
\hline Zebra fincl & : 1 msqp apvkkkrppvkeedlkgargnlaknq eiksktyqvmr qceqmgsaap sifsr artg getv fekpkdepaksvfg & 78 \\
\hline Pekin duck & msqpapvkkkrppvkeedlkgargnlsknq eiksktyqvmkqceqmg saap sifsr drtggetvfekpkdepaksvfg & 78 \\
\hline
\end{tabular}

Figure 2. Amino acid sequence encoded by the CDS of duck MUSTN1. The underlined sequences (PVKKKRPPV) are the classic nuclear import signals of the MUSTN1 gene in different species.

Seven fragments of MUSTN1 were obtained using 7 pairs of primers (Table 1 primers pMUSTN-1-7). The obtained fragments were assembled into a single contig (6435 bp; accession No. JX843528), which contained 3 exons $(9,121$, and $107 \mathrm{bp})$ and 2 introns (2261 and $846 \mathrm{bp}$ ). The exon/intron boundaries were in agreement with the GT/AG rule. The structure 
of the duck MUSTN1 gene is shown in Figure 4. In total, 231 transcription factor-binding sites were predicted with score threshold (92\%) in the 2671-bp putative promoter of duck MUSTN1 through transcription factor-binding site analysis by using Jaspar database available online (http://jaspar.genereg.net/; Supplementary file). Multiple types of transcription factors such as AP-1, PAX2, FOX family, GATA family, ETS1, HLTF, MZF1_1-4, NFIC, PDX1, PRRX2, and SPIB were found in these binding sites.

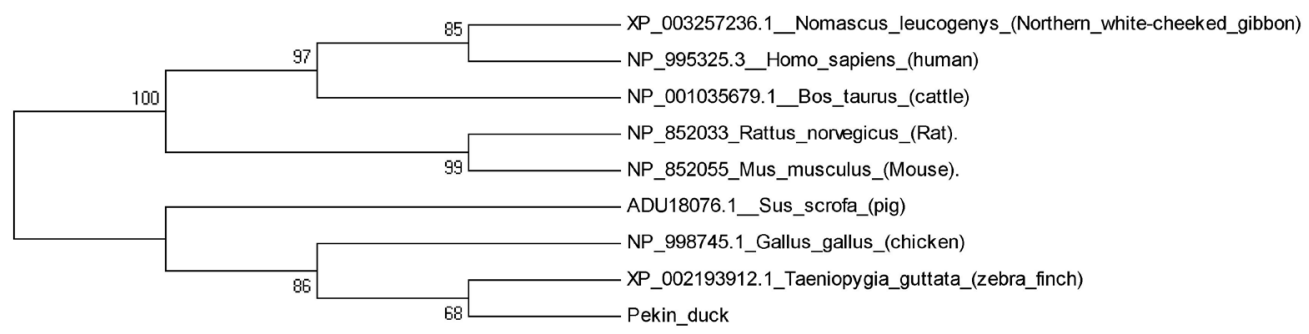

Figure 3. Phylogenetic tree of MUSTN1 amino acid sequences constructed using the MEGA software by using the neighbor-joining method and bootstrap resampling (1000 times). The predicted amino acid sequences of duck MUSTN1 and the long form MUSTN1 of other species were used in this analysis. The numbers indicate the bootstrap value (\%).

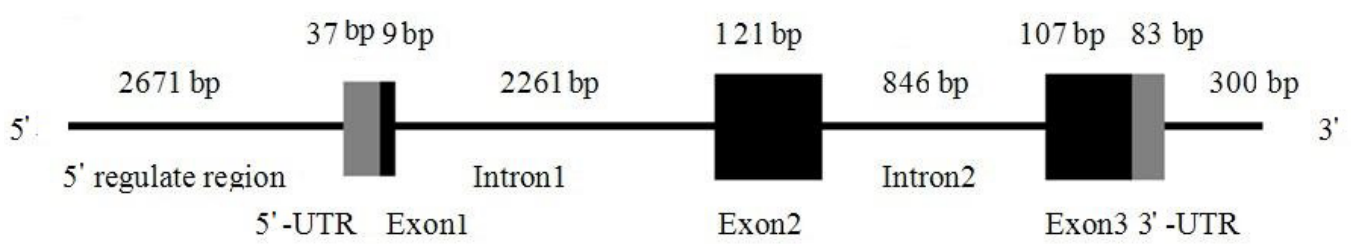

Figure 4. Genomic organization of the duck MUSTN1 gene. Numbers above each box or line represent the length of corresponding regions.

\section{Spatial and temporal expression patterns of duck MUSTN1}

qRT-PCR was performed to further investigate the spatial and temporal expressions of the duck MUSTN1 gene (Figure 5A and B). In BM, MUSTN1 expression increased from postnatal 1 week of age to 5 weeks of age and then decreased to 9 weeks of age (the relative expression increased from 2.38 to 2.76 and then decreased to 2.08; Figure 5A). The relative expression of MUSTN1 at 5 weeks of age was significantly higher than that at the other stages $(\mathrm{P}<0.05)$, while there was no significant difference in the expression level at the other stages $(\mathrm{P}>0.05)$. In LM, the expression of MUSTN1 increased from 1 week of age to 3 weeks of age and then decreased to 9 weeks of age (increased from 2.84 to 3.07 and then decrease to 2.75; Figure 5B). However, there was no significant difference in expression level among the 5 stages $(\mathrm{P}>0.05)$. At 7 weeks of age, MUSTN1 expression was the highest in the heart (5.31), which was extremely significantly $(\mathrm{P}<0.01)$ or significantly higher $(\mathrm{P}<0.05)$ than that in the other tissues. The next highest expression was noted in small intestine (2.81), tibia (2.79), and BM (2.23), while the liver (1.96), gizzard (1.78), and abdominal fat (1.63) tissues showed lower expression (Figure 6). The error bars for MUSTN1 expression in not only LM and BM but also different tissues were relatively small, suggesting that the spatial and temporal expressions of MUSTN1 can be replicated with smaller variances. 


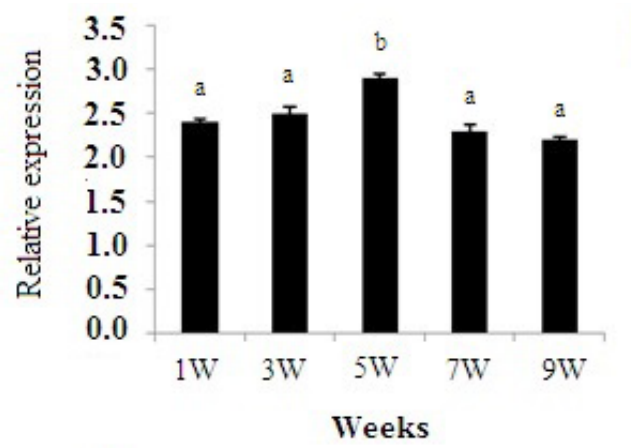

A

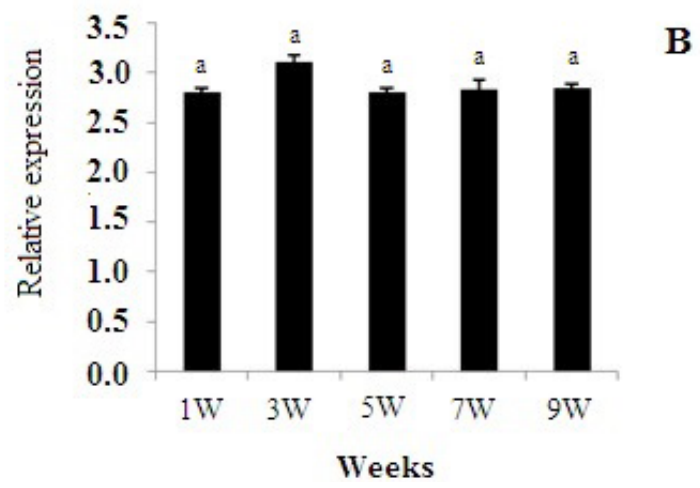

Figure 5. Relative expression level of the duck MUSTN1 gene in breast muscle (pectoralis major; A) and leg muscle (thigh; B) at different weeks of ages. $1 \mathrm{~W}, 3 \mathrm{~W}, 5 \mathrm{~W}, 7 \mathrm{~W}$, and $9 \mathrm{~W}$ present the first, third, fifth, seventh, and ninth week of age, respectively. Bars marked with the same letters denote no significant difference $(\mathrm{P}>0.05)$, and those marked with different letters denote significant difference $(\mathrm{P}<0.05)$. The relative expression level (mean value $\pm \mathrm{SD}$ ) was obtained after normalization to $\beta$-actin.

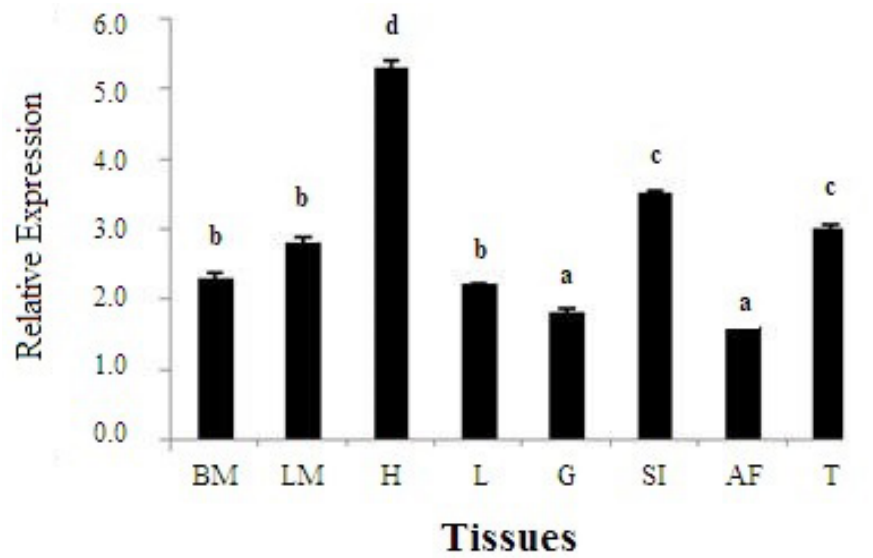

Figure 6. Relative expression level of the duck MUSTN1 gene in 8 tissues at W7. BM = breast muscle; $\mathrm{LM}=$ leg muscle; $\mathrm{H}=$ heart; $\mathrm{L}=$ liver; $\mathrm{G}$ = gizzard; $\mathrm{SI}=$ small intestine; $\mathrm{AF}=$ abdominal fat; $\mathrm{T}=$ tibia. Bars marked with the same letters denote no significant difference $(P>0.05)$, and those marked with different letters denote significant difference $(\mathrm{P}<0.05)$. The relative expression level (mean value $\pm \mathrm{SD})$ was obtained after normalization to $\beta$-actin. 


\section{Morphologic analysis of leg and breast muscle fibers}

Figures 7 and 8 show the cross-sections of LM and BM fibers, respectively, from 1 week to 9 weeks of age. LM fibers continuously developed from 1 to 9 weeks of age. However, the increase in the number of fibers from 1 to 3 weeks of age and that from 5 to 7 weeks of age was faster than that in the other weeks. The BM fibers showed a similar development process, but the growth was slower than that of LM at each stage (Figure 8).

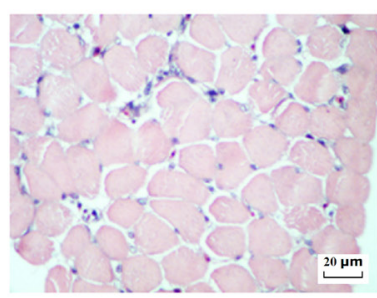

1W LM

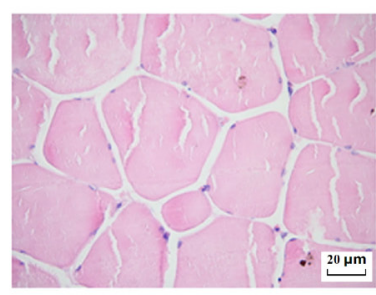

7 W LM

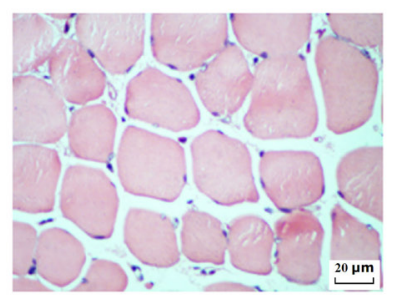

$3 \mathrm{~W}$ LM

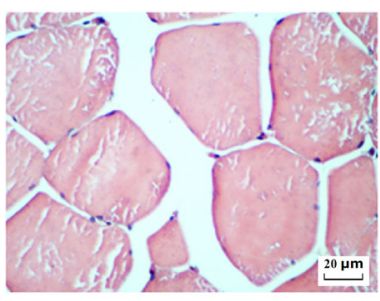

9W LM

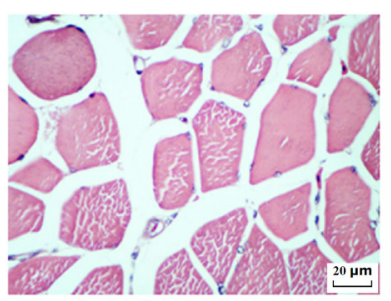

$5 \mathrm{~W} \mathrm{LM}$

Figure 7. Cross-section of leg muscle (LM) fibers (thigh) of ducks at different weeks of age.

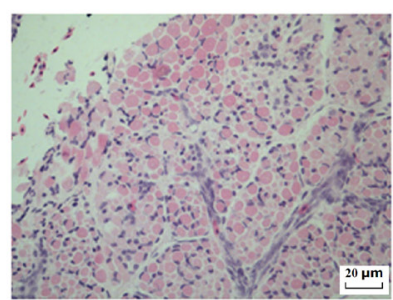

1W BM

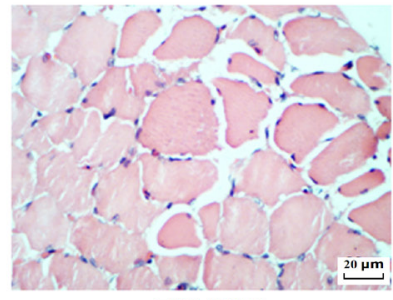

7W BM

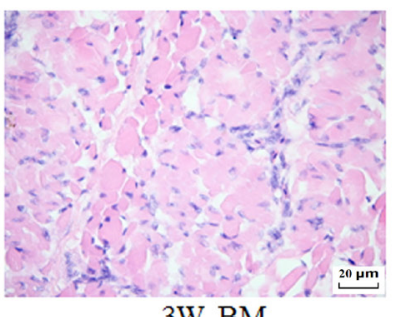

3W BM

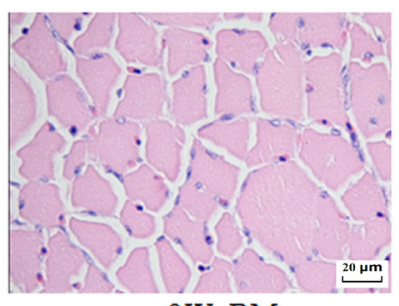

9W BM

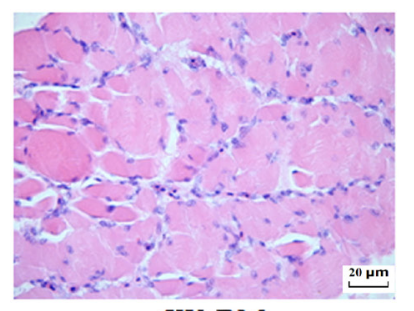

5W BM

Figure 8. Cross-section of breast muscle (BM) fibers (pectoralis major) of ducks at different weeks of age. 
The difference in the average area of muscle fibers in BM and LM at different weeks of age is shown in Figure 9. Overall, the average area of LM fibers increased more rapidly than that of BM fibers. Specifically, the average fiber area of LM was extremely significantly higher than that of $\mathrm{BM}$ at each stage $(\mathrm{P}<0.01)$. The difference in the RGR of muscle fibers in $\mathrm{BM}$ and LM at different stages is shown in Figure 10. The RGR at 3 to 5 weeks of age was the largest in BM (3.05) and that at 1 to 3 weeks of age was the largest (6.96) in LM. Generally, the RGRs of muscle fibers in BM were significantly higher than those in $\mathrm{LM}(\mathrm{P}<0.01)$. However, the RGR at 1 to 3 weeks of age in LM was significantly higher than that in $\mathrm{BM}(\mathrm{P}<0.01)$. The error bars for the average area and RGR for both LM and BM were small, indicating that the muscle size was relatively stable in the LM and BM of Pekin ducks.

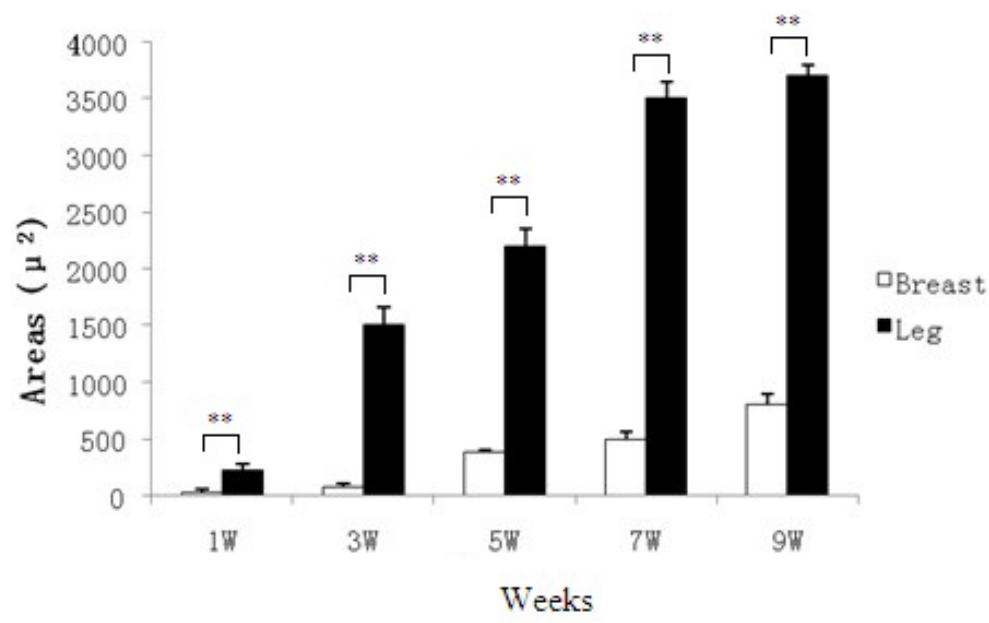

Figure 9. Average area of breast (pectoralis major) and leg muscle (thigh) fibers of ducks at different weeks of age. **Indicates extremely significant difference between the 2 bars. $\mathrm{W}=$ weeks.

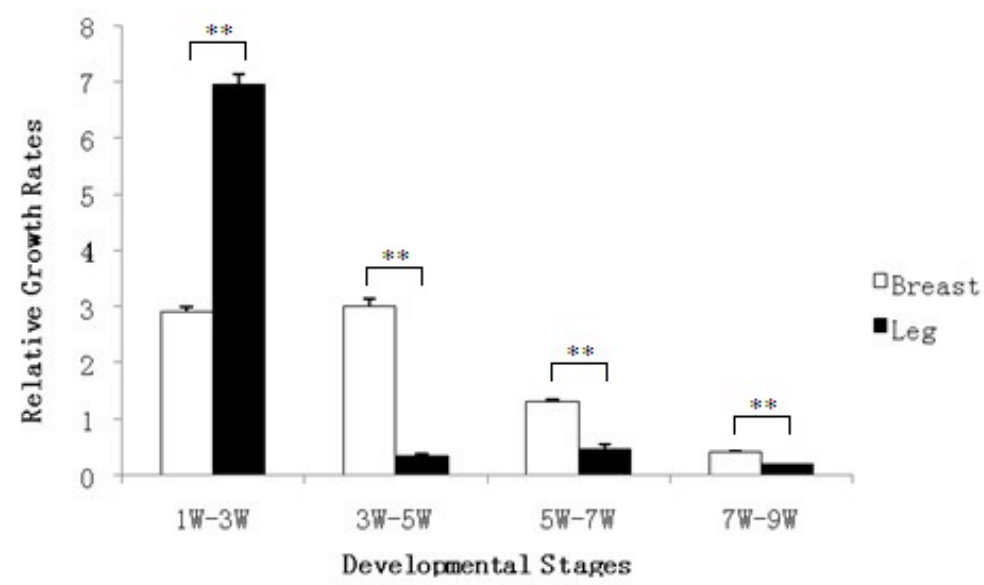

Figure 10. Relative growth rates of breast (pectoralis major) and leg muscle (thigh) fibers of ducks at different developmental ages. ${ }^{* *}$ Indicates extremely significant difference between the 2 bars. $\mathrm{W}=$ weeks. 


\section{DISCUSSION}

\section{Characteristics of the duck MUSTN1 gene}

Avian (e.g., duck, chicken, and zebra finch) MUSTN1 genes encode a 78-amino acid sequence, while mammalian (e.g., human, cattle, rat, mouse, and northern white-checked gibbon) MUSTN1 genes encode a sequence of 82 amino acids (Figure 2). This indicates the difference in genetic relationships between avian and mammalian species. Lombardo et al. (2004) described that the rat MUSTN1 amino acid sequence contained a classic nuclear import signal PIKKKRPPV, and it was most likely involved in activities other than "housekeeping". In this study, we also found a classic nuclear import signal in the duck MUSTN1 amino acid sequence. Therefore, we speculated that the duck MUSTN1 protein was a nucleoprotein involved in activities other than housekeeping. Lombardo et al. (2004) also showed that the rat MUSTN1 protein shared high similarity with those of mouse, human, and cow $(>85 \%)$. In this study, the amino acid sequence of duck MUSTN1 also showed high similarity with those of other species ( $>80 \%$; Table 2). This result indicates that MUSTN1 is evolutionarily conserved. Our bioinformatic analysis revealed that a 2671-bp region upstream of the 5'-end was probably the MUSTN1 promoter due to its classical features and multiple transcription factor-binding sites. Among the transcription factors, the AP-1 family and FOXOs are the 2 types of being identified as typical regulators of skeletal muscle development. An AP-1 factor might play an important role in the maintenance of muscle mass, and the decrease in its transcriptional activity might contribute to the loss of muscle mass in atrophic conditions (Lecker et al., 2004; Sacheck et al., 2007; Markou et al., 2008; Avouac et al., 2012). FOXOs belong to the FOX (Forkhead box) family of transcription factors. The activation of the FOXO transcription factors has been shown to be crucial in the development of muscle wasting and for the induction of atrogin-1 and MuRF-1, whose induction is essential for rapid atrophy (Bodine et al., 2001; Jagoe et al., 2002; Hasselgren, 2007). Moreover, activation of FOXO3 by itself is sufficient to cause remarkable atrophy (Glass, 2005). The above results suggest that MUSTN1 might play a critical role in skeletal muscle development.

\section{Relationship between the MUSTN1 expression level and skeletal muscle fiber morphology in Pekin ducks}

Although MUSTN1 has been reported to be expressed in skeletal muscles of rats (Lombardo et al., 2004; Liu et al., 2010), the spatial and temporal expression patterns of duck MUSTN1 have not been reported. In the present study, MUSTN1 expression in BM increased from 1 to 5 weeks of age and then decreased gradually. MUSTN1 expression in LM showed a similar trend, but the peak appeared at 3 weeks of age. MUSTN1 expression patterns in $\mathrm{BM}$ and LM are consistent with skeletal muscle development of Pekin ducks, suggesting that MUSTN1 is probably one of the regulators for Pekin duck skeletal muscle development.

In avian species, the skeletal muscle undergoes development and maturation in structure and function during incubation (Picard et al., 2002). In chicken, primary muscle fibers form at about the sixth day of hatching and secondary muscle fibers form at about 12-16 days after hatching (Stickland et al., 2004; Du et al., 2010). Differentiation of both primary and secondary fibers is complete during the 3 quarters of incubation, and the total muscle fiber numbers are therefore fixed in the final stage of avian embryos (Stockdale and Miller, 1987). 
However, the postnatal hypertrophy process subsequently increases the length and diameter of these fibers (Huang et al., 2008). Therefore, the RGR of muscle fiber area is an indirect indicator reflecting the growth rate of muscle mass in the postnatal stage. In the present study, the largest RGRs of BM and LM were those of 3 to 5 and 1 to 3 weeks of age, respectively. This indicates that the 2 periods are important in duck muscle mass increase. These results are in agreement with the patterns of skeletal muscle development in Pekin ducks. Interestingly, the peaks of MUSTN1 mRNA level in BM and LM are at 5 and 3 weeks of age, respectively (Figure 5). The above mentioned results suggest that MUSTN1 is a potential regulator of Pekin duck skeletal muscle development.

\section{ACKNOWLEDGMENTS}

We are grateful to Wei Huang, Junying Yu, and Qi Zhang (Institute of Animal Sciences, Chinese Academy of Agricultural Science, Beijing, China) for preparing of our experiment; Zhanbao Guo (Institute of Animal Sciences, Chinese Academy of Agricultural Science, Beijing, China) for data collection; and Ailing Zhang (South China Agricultural University, Guangzhou, China) and Yunsheng Zhang (Institute of Animal Sciences, Chinese Academy of Agricultural Science, Beijing, China) for reviewing the manuscript. Research supported by the Earmarked Fund for China Agriculture Research System (\#CARS-43-1, \#CARS-43-42) and the Chinese National Key Technology R\&D Program during the 11th Five-Year Plan Period (\#2006BAD14B06).

\section{REFERENCES}

Avouac J, Palumbo K, Tomcik M, Zerr P, et al. (2012). Inhibition of activator protein 1 signaling abrogates transforming growth factor beta-mediated activation of fibroblasts and prevents experimental fibrosis. Arthritis Rheum. 64: 1642-1652.

Bodine SC, Latres E, Baumhueter S, Lai VK, et al. (2001). Identification of ubiquitin ligases required for skeletal muscle atrophy. Science 294: 1704-1708.

Buckingham M, Bajard L, Chang T, Daubas P, et al. (2003). The formation of skeletal muscle: from somite to limb. $J$. Anat. 202: 59-68.

Chandra V, Kumar GS and Sharma GT (2011). Temporal expression pattern of insulin-like growth factors (IGF-1 and IGF-2) ligands and their receptors (IGF-1R and IGF-2R) in buffalo (Bubalus bubalis) embryos produced in vitro. Livest. Sci. 135: 225-230.

Draghia-Akli R, Malone PB, Hill LA, Ellis KM, et al. (2002). Enhanced animal growth via ligand-regulated GHRH myogenic-injectable vectors. FASEB J. 16: 426-428.

Du M, Tong J, Zhao J, Underwood KR, et al. (2010). Fetal programming of skeletal muscle development in ruminant animals. J. Anim. Sci. 88: E51-E60.

Glass DJ (2005). Skeletal muscle hypertrophy and atrophy signaling pathways. Int. J. Biochem. Cell Biol. 37: 1974-1984.

Gu Z, Zhang Y, Shi P, Zhang YP, et al. (2004). Comparison of avian myostatin genes. Anim. Genet. 35: 470-472.

Hadjiargyrou M, Lombardo F, Zhao S, Ahrens W, et al. (2002). Transcriptional profiling of bone regeneration. Insight into the molecular complexity of wound repair. J. Biol. Chem. 277: 30177-30182.

Hasselgren PO (2007). Ubiquitination, phosphorylation, and acetylation-triple threat in muscle wasting. J. Cell Physiol. 213: 679-689.

Huang TH, Zhu MJ, Li XY and Zhao SH (2008). Discovery of porcine microRNAs and profiling from skeletal muscle tissues during development. PLoS One 3: e3225.

Jagoe RT, Lecker SH, Gomes M and Goldberg AL (2002). Patterns of gene expression in atrophying skeletal muscles: response to food deprivation. FASEB J. 16: 1697-1712.

Kanisicak O, Mendez JJ, Yamamoto S, Yamamoto M, et al. (2009). Progenitors of skeletal muscle satellite cells express the muscle determination gene, MyoD. Dev. Biol. 332: 131-141.

Lecker SH, Jagoe RT, Gilbert A, Gomes M, et al. (2004). Multiple types of skeletal muscle atrophy involve a common program of changes in gene expression. FASEB J. 18: 39-51. 
Lee SJ and McPherron AC (1999). Myostatin and the control of skeletal muscle mass. Curr. Opin. Genet. Dev. 9: 604-607. Li L, Liu HH, Xu F, Si JM, et al. (2010). MyoD expression profile and developmental differences of leg and breast muscle in Peking duck (Anas platyrhynchos Domestica) during embryonic to neonatal stages. Micron 41: 847-852.

Liu C and Hadjiargyrou M (2006). Identification and characterization of the Mustang promoter: regulation by AP-1 during myogenic differentiation. Bone 39: 815-824.

Liu C, Gersch RP, Hawke TJ and Hadjiargyrou M (2010). Silencing of Mustn1 inhibits myogenic fusion and differentiation. Am. J. Physiol. Cell Physiol. 298: C1100-C1108.

Liu HH, Wang JW, Li L, Han CC, et al. (2011). Molecular evolutionary analysis of the duck MYOD gene family and its differential expression pattern in breast muscle development. Br. Poult. Sci. 52: 423-431.

Livak KJ and Schmittgen TD (2001). Analysis of relative gene expression data using real-time quantitative PCR and the 2(-Delta Delta C(T)) method. Methods 25: 402-408.

Lombardo F, Komatsu D and Hadjiargyrou M (2004). Molecular cloning and characterization of Mustang, a novel nuclear protein expressed during skeletal development and regeneration. FASEB J. 18: 52-61.

Maiti D, Xu Z and Duh EJ (2008). Vascular endothelial growth factor induces MEF2C and MEF2-dependent activity in endothelial cells. Invest. Ophthalmol. Vis. Sci. 49: 3640-3648.

Markou T, Cullingford TE, Giraldo A, Weiss SC, et al. (2008). Glycogen synthase kinases $3 \alpha$ and $3 \beta$ in cardiac myocytes: regulation and consequences of their inhibition. Cell Signal. 20: 206-218.

Picard B, Lefaucheur L, Berri C and Duclos MJ (2002). Muscle fibre ontogenesis in farm animal species. Reprod. Nutr. Dev. 42: 415-431.

Sacheck JM, Hyatt JP, Raffaello A, Jagoe RT, et al. (2007). Rapid disuse and denervation atrophy involve transcriptional changes similar to those of muscle wasting during systemic diseases. FASEB J. 21: 140-155.

Sambrook J and Russell D (2001). Molecular Cloning: A Laboratory Manual. 3rd edn. Cold Spring Harbor Laboratory, Cold Spring Harbor.

Stickland NC, Bayol S, Ashton C and Rehfeldt C (2004). Manipulation of Muscle Fibre Number During Prenatal Development. In: Muscle Development of Livestock Animals (Te Pas MFW, Everts ME and Haagsman HP, eds.). Physiology, Genetics, and Meat Quality, Wallingford, 70-71.

Stockdale FE and Miller JB (1987). The cellular basis of myosin heavy chain isoform expression during development of avian skeletal muscles. Dev. Biol. 123: 1-9.

Wu Y, Liu XL, Xiao HW, Wang J, et al. (2009). Expression pattern and prokaryotic expression for peking duck insulinlike growth factor-I in Escherichia coli. Biochem. Genet. 47: 802-811.

Xu TS, Zhang XH, Gu LH, Zhou HL, et al. (2012). Identification and characterization of genes related to the development of skeletal muscle in the Hainan black goat. Biosci. Biotechnol. Biochem. 76: 238-244. 